\title{
Miradas olvidadas: la vida cotidiana de docentes de principios del siglo XX
}

\author{
Fernando Sandoval Gutiérrez
}

Universidad Autónoma de Ciudad Juárez

DM Cuauhtémoc

\section{Resumen}

1 jo y a la vida cotidiana de las y los profesores de las escuelas primarias en el estado de Chihuahua en la primera década del siglo XX. El texto se construyó con material acopiado en el Archivo de Concentración del Gobierno del Estado. Se plantea la visión de que los profesores y profesoras en este periodo vivían condiciones específicas de trabajo asociadas al desarrollo de la última época del Porfiriato en la entidad y a la gestación del movimiento revolucionario. Finalmente se destaca el valor que los expedientes de servicio - en especial el material fotográfico- tienen para al estudio de la historia de la educación chihuahuense.

Palabras clave: historia de la práctica docente, vida cotidiana escolar, historia de la educación en Chihuahua

\section{Introducción}

El gobierno del estado de Chihuahua conserva un amplio registro documental de la operación de sus diversas dependencias en su archivo de concentración. Ahí se conservan los expedientes de los profesores chihuahuenses desde 1880 hasta finales del siglo pasado en miles de legajos que conservan las historias profesionales de quienes ejercieron la docencia al servicio de la administración estatal. Los expedientes ofrecen un contenido adicional de gran valor: muchos de ellos contienen fotografías originales de los profesores, incluidas por razones administrativas en diversos documentos oficiales, especialmente en las hojas de servicio que se acopiaban periódicamente. Revisarlos permite conocer cara a cara a los protagonistas de las historias que aparecen al leer los antiguos oficios y cartas. 
En las páginas siguientes ofrecemos una visión de diversas dimensiones de lo cotidiano para estos maestros y maestras en Chihuahua antes del inicio del movimiento revolucionario de 1910 . Se trata de una visión construida a partir de los primeros hallazgos de la exploración de los expedientes de personal, que aún aguardan una revisión exhaustiva.

\section{La vida en sepia}

El siglo XX empezó en el estado de Chihuahua trayendo consigo una avalancha de cambios. El primero y más importante fue una relativa vuelta a la tranquilidad perdida muchos años atrás asociada a las guerras indígenas del siglo XIX (Orozco, 1992). Durante esos primeros años del nuevo siglo, Chihuahua se serenó. La entidad poco a poco se iba "civilizando" según se afirmaba en un periódico de ese entonces, sus páginas se llenaban con chabacanas noticias del robo de una bicicleta, o de la pérdida de un par de medias de seda de uno de los almacenes de la ciudad (El Correo de Chihuahua, 1906). La prueba máxima de ese alejamiento de la barbarie lo constituían los nuevos adelantos tecnológicos: primero el ferrocarril a Juárez y a la ciudad de México en 1882, dos años después el teléfono y por esas mismas fechas la luz eléctrica, hechos que asombraron a los chihuahuenses (Dale Lloyd, 1987). Poco después llegarían hasta Chihuahua las máquinas de coser Singer, los primeros automóviles y aparatos eléctricos de aire acondicionado (Idem, 2001).

Los progresos del estado se reflejaban en su capital. La ciudad de Chihuahua se había convertido en una urbe que rondaba los sesenta mil habitantes, marcada por el refinamiento arquitectónico; a la vieja catedral con sus adornos barrocos ahora le hacían compañía numerosos edificios: el Instituto Científico y Literario, el Palacio de Gobierno, el Hospital Porfirio Díaz y el flamante Teatro de los Héroes, con sus enormes paredes coloradas (Instituto Nacional de Estadística y Geografía, 2009). El desarrollo explosivo de la capital se reflejó en su desdoblamiento espacial por medio de la fundación de nuevas colonias, por esas fechas se pusieron los cimientos de las primeras casas en el Santo Niño, en el barrio del Pacífico y en el rumbo de la Penitenciaría. En 1910, el erudito José María Ponce de León propuso comprender la anatomía de la ciudad usando como herramienta la figura de un pentágono: la base del dibujo correspondía al río Chuvíscar, uno de sus bordes laterales se formaba desde la junta de los ríos, donde estaba la estación del tren, hasta el cerro Coronel, y el otro desde el Santuario de Guadalupe hasta el Cerro Grande. El último lado se formaba entre los dos cerros. Por ahí, escribió Ponce de León (1907) "se asoma la ciudad, como muchacha coqueta".

El crecimiento de los asentamientos humanos afectó profundamente la vida cotidiana. Por ejemplo, el uso de los tiempos libres se modificó de manera drástica en el transcurso de apenas diez años; entre 1900 y 1910 Chihuahua conoció el cinematógrafo, el fonógrafo, y diversos ritmos musicales norteamericanos y europeos (Balderrama Montes y Pérez Galindo, 1999). Los cambios en la vida de todos los días se complementaron con la llegada de muchos 
productos nuevos. Por la vía del tren comenzaron a llegar a Chihuahua alimentos enlatados, refrescos, revistas y libros, géneros multicolores, joyas de fantasía, relojes, y otros artículos (Lau, 1992). También en tren llegaron al estado hombres y mujeres de muchas latitudes, atraídos por las posibilidades de trabajo; entre 1890 y 1910 se fundaron o reabrieron cincuenta y cuatro minerales en Chihuahua (Canudas, 2005) que junto con las grandes propiedades agropecuarias y la industria maderera serrana se convirtieron en polos de atracción de inmigrantes.

En ese marco, la actividad educativa en las escuelas chihuahuenses se presentaba pujante. A pesar de las contradicciones sociales y económicas presentes en el estado, que originarían el estallido del movimiento revolucionario en Chihuahua en 1910, en los salones de clase de la entidad se presentaban procesos bien interesantes de desarrollo educativo.

\section{Los nuevos profesores}

En 1905, Rómulo Escobar, uno de los dos célebres hermanos que en ese año fundaron la Escuela de Agricultura en ciudad Juárez, recomendó a los lectores del periódico que editaba:

Saquen ustedes un maestro de escuela de cualquier parte, al azar, pepénenlo del montón, sin escogerlo; cualquiera es igual a los otros y pónganselo en la palma de la mano. Fíjense bien en él. Está flaco el pobre, triste y meditabundo, con el brillo de la vista apagado y la dispepsia en el estómago, tiene que gastar él solo toda la bilis que deberíamos gastar los padres de los chamacos (Escobar y García García, 2005).

Además de sus problemas biliosos, los maestros chihuahuenses de los primeros años del siglo XX tenían que lidiar con otros conflictos. El primer obstáculo era el de convertirse en maestro. Como un efecto de la profesionalización del servicio educativo, las "casas de amigas", establecimientos de educación informal instaladas en domicilios particulares en donde se repasaba el silabario de San Miguel tendieron a desaparecer (Coromias, 2014). El avance de la institucionalización del servicio educativo y la asunción del gobierno como instancia monopolizante de la oferta educativa, generaron un proceso de normalización del acceso al servicio docente.

De todas maneras para aquél que decidiera aventurarse por los caminos de la enseñanza, estaba bien claro que no le esperaba un futuro de holganza económica: nadie se hacía rico enseñando. Al contrario, los profesores con frecuencia se veían en la necesidad de emplearse en otras actividades alejadas de la docencia para poder completar el gasto familiar. En esta época inició también en el gremio de los maestros la práctica de endeudarse a cuenta de salarios futuros para obtener de los comerciantes artículos y servicios de diversa naturaleza (Gobierno del Estado de Chihuahua, 1906). Es llamativo que en los expedientes de algunos maestros chihuahuenses de finales del siglo XIX todavía se pueden leer antiguos pagarés por la entrega de la leche semanal, o por un juego de sala, junto con las reiteradas notas de los tenderos para que los profesores pagaran (Gobierno del 
Estado de Chihuahua, 1906). Estamos hablando además de una época en la que los profesores de Chihuahua no se habían organizado como gremio por lo que no contaban con alternativas de financiamiento o préstamos de ningún tipo además de las que ofrecían las instituciones bancarias instaladas en el estado por esas fechas.

\section{La jornada escolar}

Aunque no existía una reglamentación clara que normara los procesos educativos a finales del siglo XIX, la rutina escolar tendía a grandes rasgos hacia la homogeneidad (Larios y Hernández, 2012). Ello en el marco de salones de clase no siempre adecuados a las necesidades de los educandos; Ignacio Manuel Altamirano (1970) en Beatriz, uno de sus de sus cuentos inconclusos, dice que algunos de los salones en los que trabajaban los alumnos eran magníficos, pues se hallaban modelados según los aposentos que se destinaban a los criminales en las cárceles de aquel tiempo.

Luego de un primer lapso de estudio, generalmente se concedía a los estudiantes entre quince minutos y media hora de recreo, en la que los niños jugaban a la pelota, a los huesos de chabacano, al trompo, o bien a "el diablo y la monja", para luego continuar con la jornada escolar que normalmente se volvía a interrumpir cerca de las doce del día para la comida. Lo común era que las actividades escolares matutinas se complementaran con jornadas por la tarde, de dos o tres horas diarias (Gobierno del Estado de Chihuahua, 1906).

En muchos casos el profesor aparecía como un elemento eminentemente puniti- vo de las conductas presuntamente inmorales o libertinas de sus alumnos. Un sufrido ex-alumno de la época recordaba en su adultez que en la cátedra, un profesor lleno de sabiduría nos explicaba el texto y nos ponía de rodillas de cuando en cuando, si no sabíamos la clase, o bien nos hacía encerrar en un cuarto un rato o nos pegaba en el trasero con una varita de membrillo.

En otras escuelas el castigo era todavía más enérgico, se aplicaban al alumno vago, cualesquiera que fuera su edad, sendos golpes con una palmeta (Gobierno del Estado de Chihuahua, 1906). En muchos casos el precepto de la letra con sangre entra se aplicaba tal cual. El uso de la palmeta para castigar a quien observaba mala conducta o no demostraba aplicación, o el empleo de las agujas o el dedal para las clases de bordado, que en ocasiones servía para corregir la falta de talento de las menores, o los métodos pedagógicos que se basaban en la memorización y no en la comprensión, así como las zurras combinadas de los profesores y los padres de familia, fueron el eje de la educación moral y religiosa durante gran parte del siglo XIX y de principios del XX en Chihuahua y en muchas otras latitudes (Robles, 1997).

Este énfasis de los profesores en la aplicación de sanciones diversas formaba parte importante del currículo oculto y estaba en concordancia con las propuestas curriculares de la época que se cimentaban en la idea de que la disciplina era la madre del aprendizaje efectivo, la base del carácter y los buenos modales. También tenían que ver con la conformación del rol social del profesor al interior de la sociedad norte- 
ña, como un proveedor de conocimientos pero también de promotor de un conjunto de valores prescritos para los niños y socialmente legitimado por los chihuahuenses (Robles, 1997).

El aprendizaje de la lectura y la escritura estaban en primer lugar de prioridades. Se enseñaba a leer, escribir y las cuatro reglas de cuentas. Algunas escuelas agregaban clases de dibujo y de manualidades para las niñas, aunque este plan de estudios sufría modificaciones bien amplias de acuerdo a los tiempos, a la formación de los profesores, y a los libros de texto que se encontraban a la mano. Frecuentemente se hacía mucho hincapié en la formación valoral de los niños, casi siempre mediante fábulas (Gobierno del Estado de Chihuahua, 1906).

En los años superiores de la primaria las materias se complicaban. Se enseñaba entonces gramática latina, por Nebrija o Iriarte, para luego pasar al estudio de la lógica por Jaquier, por Bouvier o por Heineccius. En cuanto a la educación física, en Chihuahua no se conoció a un maestro especializado en esa materia sino hasta 1906. Antes se practicaba la gimnasia sueca, usando garrotes y pompones para realizar diversas ejecuciones, y los ejercicios militares con rifles de palo y combates ficticios organizados por los propios docentes (Gobierno del Estado de Chihuahua, 1906).

\section{Las escuelas}

Los planteles escolares de principios del siglo XX estaban marcados por la heterogeneidad. La necesidad de muchos padres de familia en el sentido de que sus hijos tuvieran la oportunidad de experimentar una formación escolar generó la creación o adaptación de numerosos espacios que se consagraron a las actividades educativas (Gobierno del Estado de Chihuahua, 1906). En muchos casos se trataba tan solo de edificaciones originalmente pensadas como casas habitación que se acondicionaban para recibir a los niños y a sus maestros; en otros casos, los menos, las escuelas eran cuidadosamente planeadas y construidas por arquitectos especializados, con base en las últimas prescripciones higiénicas y pedagógicas de la época.

En medio de estos dos extremos podían encontrarse escuelas instaladas en las más variadas condiciones: casas de hospedaje acondicionadas como centros, anexos de edificios públicos, bodegas; prácticamente cualquier lugar que pudiera albergar a un grupo de niños y a su profesor era bueno. Las áreas de juego eran amplias y se procuraba que en las escuelas en las que se ofrecía servicio a niños y a niñas estuvieran divididas por sexo. Estas áreas servían también para la realización de diversas actividades cívicas. Las canchas deportivas hicieron su aparición en las escuelas de Chihuahua poco después.

$\mathrm{El}$ arquetipo de esta manera de entender los centros educativos fue materializado en 1906, con la construcción de la escuela No. 141, en la esquina de la avenida Penitenciaría (hoy 20 de Noviembre) y Terrazas de la ciudad de Chihuahua. La construcción original de este plantel refleja las nociones que se consideraban ideales para la práctica educativa y sirvió de modelo a edificaciones sucesivas: un edificio central, compuesto por un salón de actos con altos techos 
y pisos de madera y a su alrededor largos corredores que daban acceso a las aulas; todo ello rodeado por dos áreas de juego (Sandoval Gutiérrez, 2003). Una excepción en la austeridad reinante en el equipamiento de las escuelas la constituían los centros educativos de la Sociedad Filomática instalados en la ciudad de Chihuahua. Estos planteles los fundaron grupos de padres de familia pudientes, con el propósito de asegurar que sus hijos pudieran vivenciar procesos educativos de alta calidad. Estas escuelas fueron equipadas trayendo de El Paso, Texas, lo último en menaje y material escolar (Sociedad Filomática de Chihuahua, México, 1908).

\section{Los niños y las niñas}

Del otro lado de la moneda educativa aparecían los niños chihuahuenses. A pesar de que el paradigma escolar dominante en ese entonces promovía la imagen del profesor como el protagonista de lo que ocurría al interior de las aulas, entonces como hoy, los verdaderos personajes centrales de la realidad educativa eran los niños y las niñas que llenaban los salones de clase.

Ser niño en el estado de Chihuahua a principios del siglo pasado, implicaba vivir la infancia en un mundo que ofrecía amplios espacios para relacionarse con la naturaleza, para aprender a partir de la experiencia, pero que también implicaba el enfrentamiento con rígidas posiciones de la mayor parte de los adultos con respecto a cómo deberían educarse los infantes.

Parte de esta rigidez se mostraba en un interés por reconocer a los mejores. En 1906 el Gobernador Enrique Creel ordenó que de manera mensual los centros educativos reportaran a la estructura educativa los nombres de los alumnos con mejores desempeños para integrar una lista de honor que se publicaba en la gaceta oficial. Esta medida generó pugnas bien intensas por ocupar los lugares de privilegio en esa lista, que significaba la exhibición pública de los méritos académicos de los niños (Mayer, 2007).

Los niños tenían otras alternativas para alcanzar la atención de la gente. Eran especialmente apreciados por la comunidad chihuahuense los cuadros gimnásticos, que se practicaban con indumentarias especiales y que consistían en la ejecución bien precisa de diversos movimientos y ejercicios por parte de grupos numerosos de alumnos (Gobierno del Estado de Chihuahua, 1906). Estas ejecuciones sé preparaban para algún evento especial, la conmemoración de alguna fecha histórica de importancia o con motivo de la visita de un personaje ilustre. Los cuadros gimnásticos se inspiraban en obras literarias protagonizadas por duendecillos o hadas mágicas o por dragones de hocico llameante y eran acompañados con frecuencia por piezas de oratoria, disciplina que era cultivada con ahínco en muchas primarias y que se consideraba como una cualidad muy importante (Gobierno del Estado de Chihuahua, 1906). Todas estas expresiones del arte desplegado por los niños chihuahuenses se desarrollaron en toda su magnitud en la visita del Presidente Porfirio Díaz al estado, apenas unos pocos meses antes del estallido de la revolución. Para esa fecha se organizó en la capital la "Fiesta de la Niñez Chihuahuense", que fue celebrada en el Teatro de 
los Héroes. Ahí, Díaz disfrutó de los alcances artísticos de los alumnos y alumnas de las escuelas primarias de la ciudad (Ayuntamiento de Chihuahua, 1993).

Para los alumnos menos afectos al movimiento corporal existía una opción más: la participación en cuadros estáticos que consistían en composiciones vivientes acerca de un tema específico: las fuerzas naturales, una escena histórica, algún tópico que se pretendía explicar mediante su representación visual, una especie de performance temprano que casi siempre era fotografiado para la posteridad. Estos cuadros estáticos servían adicionalmente para hacer públicas las habilidades para las artes manuales de los docentes, que se encargaban de la confección de los materiales.

En realidad la escuela dejaba poco tiempo libre a los niños. La disposición discontinua de los horarios de clase mantenía a los alumnos en los planteles educativos ocupados en actividades de muy diversa índole, mucho tiempo más que a los niños de la actualidad. Más allá de las puertas de la escuela quedaba un mundo por explorar. Por otro lado, no eran pocos los niños y niñas del campo y la ciudad que complementaban su actividad escolar con el trabajo, generalmente en auxilio de las actividades de los padres. Esto era especialmente frecuente en el campo, sobre todo en las temporadas en las que los ciclos agrícolas exigían el trabajo de cuantas manos estuvieran disponibles. El concepto del trabajo infantil como una práctica reprochable no llegaría a Chihuahua hasta muchos años después. Estamos hablando además de una época en la que la distinción asociada al género como un procedimiento selectivo para decidir quién podía ir a la escuela y quién se quedaba en casa o en el trabajo era mucho más importante que hoy en día. En las estadísticas de inscripción de las escuelas de la época casi invariablemente el número de niños supera al de las niñas (Gobierno del Estado de Chihuahua, 1906).

\section{Los materiales para enseñar}

Desde los años finales del siglo XIX las profesoras y profesores chihuahuenses tenían a su disposición diversos tipos de textos: cuadernos de lecciones, que contenían unidades subtemáticas pequeñas que podían abordarse en una clase; libros de ejercicios, con cuestionarios y otras actividades; libros de lectura y textos de consulta (Gobierno del Estado de Chihuahua, 1906).

Los cuadernos de lecciones ofrecían temas, frecuentemente numerados, estructurados en pequeñas módulos de una o dos páginas que podían estudiarse durante una jornada. Típicamente la lección contenida en estos libros empezaba con una definición de sus conceptos clave para luego revisar algunos significados de nociones complementarias al tema principal. En la mayor parte de los cuadernos de lecciones se recomendaba que al terminar de revisar el texto, se pasara al cuaderno de actividades o a los ejercicios en el pizarrón. De ahí que las editoriales que surtían a los profesores chihuahuenses de la época acompañaran a los libros de lecciones con abundantes cuadernos de ejercicios, que se vendían en paquete (Gobierno del Estado de Chihuahua, 1906).

Estos materiales se complementaban con textos de referencia. Este tipo de libros 
se utilizaba frecuentemente como referencia para la planeación de las clases que el profesor dictaría a sus alumnos, y ocasionalmente los mismos niños tenían acceso a estos libros para buscar información o bien para repasar los contenidos abordados en clase. Otros textos que eran de uso cotidiano en las escuelas: los "alfabetos" (científico, de artes y oficios, de juegos de la infancia, de juegos de la juventud, alfabeto mitológico, alfabeto zoológico) colecciones de fichas de cartoncillo sobre diversos tópicos; el "Amigo de los niños, escrito en francés por Sabatie y traducido hacia 1880 por Escoiquiz; la "Aritmética" de Navea; una amplia variedad de catecismos (histórico, de Ripalda, de economía doméstica, de geografía universal, de urbanidad, de perseverancia) y un buen número de compendios: de los derechos, de la urbanidad, etcétera, todos ellos junto a los textos para la enseñanza de la moral y las buenas costumbres, como el "Manual del buen tono" o el "Manual de urbanidad y de buenas maneras" de Urcullu (Benso, 1997).

En la ciudad de Chihuahua, los profesores podían conseguir estos materiales en la librería y papelería "Pluma y Lápiz", propiedad de Eugenio de la Torre. Otro recurso era pedirlos por correo a la ciudad de México, en donde se editaban e imprimían la mayoría de los materiales de apoyo para profesores, en casas libreras como "Herrero Hermanos Sucesores", "Librería de la viuda de Ch. Bouret”, “Tipografía de Aguilar e Hijos"y otras (Castañeda, 2006).

Ocasionalmente los libros contenían, además de las actividades de ejercitación de las habilidades memorísticas, ejerci- cios llamados ideológicos, que consistían en preguntas que motivaban procesos reflexivos en los alumnos. Otro clásico de la época era "El lector católico mexicano", que contenía narraciones acompañadas de viñetas en blanco y negro -salvo la ilustración de la portada, que era en todos los colores- que iban hilvanando una historia con conceptos de biología, religión o historia. Los contenidos de las lecciones no se agrupaban bajo ningún criterio, de manera tal que cada tomo de "El lector católico" era una sabrosa ensalada temática. Algunas lecciones incluían al final preguntas sobre su contenido, bajo el nombre de "ejercicios de lenguaje", y en otras más se recomendaba al profesor narrar a sus alumnos el final de una fábula, o bien pedirles que lo hicieran ellos mismos (Cadena, 1903).

\section{La realización personal}

Surgida en condiciones difíciles, la profesión magisterial en el estado de Chihuahua suponía la asunción de diversos supuestos. Uno de ellos era su potencial para que las personas que decidían convertirse en profesores o en maestras pudieran alcanzar un grado de realización personal que desde luego tenía que ver primariamente con el tema del sustento, pero que también operaba en otros niveles. De entrada los profesores y las maestras recibían tratamientos especiales por parte de la sociedad chihuahuense de entonces; las maestras eran tratadas de "señoritas", mientras que los profesores siempre eran llamados "maestros" (Gobierno del Estado de Chihuahua, 1906). Estos formulismos revelan por sí mismos elementos simbólicos bien poderosos, asociados a la autoridad, el respeto, la sabidu- 
ría. Ser profesor implicaba tener un punto de vista informado, ser considerado por la comunidad como un miembro importante, estratégico en más de un sentido.

La profesión también traía consigo una imagen que resultaba gratificante para muchos. El canon de la formalidad actuaba como un regulador de la figura de los profesores hacia la sociedad pero también como un elemento simbólico de autoafirmación para los docentes.

Otra dimensión de la realización personal tenía que ver con el apostolado magisterial incipiente que ya se gestaba por aquellos años. Los profesores eran los mensajeros del progreso, los fundadores de la civilización, los portadores de la luz del conocimiento y su influencia educativa y cultural fue determinante para el derrotero de los destinos de nuestro estado. En la vida de muchas localidades rurales del estado hicieron su aparición las kermesses, las fiestas cívicas y las graduaciones; la vida cotidiana de las localidades que contaban con un profesor se modificó sustancialmente, al grado que en algunos lugares las fiestas de la escuela desplazaron a las celebraciones tradicionales (Gobierno del Estado de Chihuahua, 1906).

La importancia que los profesores tenían para la sociedad chihuahuense era apoyada por la postura de las autoridades estatales al respecto. En los primeros años del siglo XX fueron varios los profesores que recibieron medallas y diplomas de reconocimiento al mérito académico y laboral por iniciativa del propio Gobernador del estado (Gobierno del Estado de Chihuahua, 1906).
Si el reconocimiento público de la labor de los maestros era considerable y la valoración que se hacía de su labor era muy alta, no es posible decir lo mismo de sus emolumentos. Una lista de compras de un profesor de la ciudad de Chihuahua que se encuentra en un expediente de 1909 incluye los siguientes productos: harina, chile, cominos, una caja de sal, una veladora, un frasco de vinagre, almidón, un bote de polvo para limpiar trastos, una caja de jabón y un paquete de cigarros (Gobierno del Estado de Chihuahua, 1906).

En otro sentido es interesante observar como infortunadamente, los primeros años del surgimiento de lo que sería el sistema educativo estatal también son los años de la emergencia de problemas serios: en varias partes de Chihuahua aparecen casos de profesores con serios problemas de alcoholismo, tal vez asociados a las condiciones de soledad y marginación en las que muchos desempeñaban su labor. Aparecen también problemas de salud relacionados con cuadros neuróticos, estrés, problemas en la garganta y otros malestares (Gobierno del Estado de Chihuahua, 1906). Había otras dificultades. No existía un servicio médico establecido para los trabajadores de la educación, los gastos relacionados con la atención de la salud de los maestros corrían por su cuenta y los medicamentos eran un gasto aparte.

\section{El final de la jornada}

Los profesores chihuahuenses de principios del siglo XX transitaban por el ejercicio de la docencia con diversos ritmos, y 
con destinos mucho más inciertos que los de los profesores de la actualidad. No había límites cronológicos para ejercer como profesor o profesora. Los docentes no tenían una edad establecida para jubilarse o pensionarse porque no se había implementado en ese entonces ningún servicio de pensiones, aunque generalmente el gobierno del estado atendía los gastos de pensión de los profesores, esta disposición estaba sujeta al arbitrio de los administradores (Gobierno del Estado de Chihuahua, 1906).

La comparación de la serie de fotografías de un mismo profesor, que inicia con la imagen adjunta a la hoja de ingreso al servicio docente y que se cierra con la fotografía pegada a la carta testamentaria, permiten atisbar en alguna medida los abismos profundos de su personalidad y las peculiaridades de la Chihuahua que le tocó enfrentar. Es una verdadera ventana al pasado a través de los ojos de un maestro.

Tener la oportunidad de ver a los ojos a estos maestros y maestras que actuaron en la Chihuahua de hace cien años es un verdadero privilegio. Nos permite acercarnos un poco a su vida cotidiana y a comprender la magnificencia de sus esfuerzos personales y colectivos para construir un mundo mejor a través de su labor profesional. Las fotografías y expedientes de los profesores mostrados plantean adicionalmente otros retos relacionados con la calidad del servicio educativo que hoy se ofrece en las escuelas de nuestra entidad. ¿Habrán mejorado los servicios educativos en un siglo? ¿Qué opinarían al respecto los maestros y las maestras que aparecen en este trabajo?

\section{Bibliografía}

Altamirano, I. (1970). Clemencia, cuentos de invierno, Julia, Antonio, Beatriz, Atenea. México: Porrúa.

Alvarez, M. (1960). La educación en Chihuahua: estudio histórico para las bodas de oro de la Normal de Chihuahua. México.

Ayuntamiento de Chihuahua. (1993). Visita a Chihuahua del Señor Presidente General don Porfirio Díaz. Octubre de 1909. Chihuahua: Ayuntamiento de Chihuahua.

Balderrama, R., y Pérez, R. (1999). La música en Chihuahua. 1890-1940. Chihuahua: Universidad Autónoma de Chihuahua.

Benso, C. (1997). Controlar y distinguir: la enseñanza de la urbanidad en las escuelas del siglo XIX. Vigo: Servicio de Publicacións da Universidad de Vigo.

Cadena, L. (1903). El lector católico mexicano: método de lectura conforme con la inteligencia de los niños: Libro segundo, basado en las lecciones de cosas, en la religión católica, en la sana moral : y los rigurosos cánones preceptuados por la moderna ciencia educativa. Herrero Hermanos.

Canudas, E. (2005). Las venas de plata en la historia de México. México: Universidad Juárez Autónoma de Tabasco.

Castañeda, J. (2006). La enseñanza de la Geografía en México, una visión histórica 1821-2005. México: Plaza y Valdés.

Coromias, F. (2014). Educar hoy. Madrid: Ediciones palabra.

Dale Lloyd, J. (1987). El proceso de modernización capitalista en el noroeste de Chihuahua (1880-1910). México: Universidad Iberoamericana.

Dale Lloyd, J. (2001). Cinco ensayos de cultura material de rancheros y medieros del noroeste de Chihuahua, 1886-1910. México: Universidad Iberoamericana.

El Correo de Chihuahua (1906, Octubre 8). Se roban una bicicleta. El Correo de Chihuahua, p. 2.

Escobar, R., y García, J. (2005). Don Rómulo Escobar; artículos y ensayos (1896-1946). Ciudad Juárez: Municipio de Juárez.

Gobierno del Estado de Chihuahua. (1906). Archivo de Concentración de la Administración Estatal. Fondo expedientes de personal de educación. Chihuahua: Gobierno del Estado de Chihuahua.

Instituto Nacional de Estadística y Geografía. (2009). Estadísticas históricas de México. México: INEGI.

Larios, M., y Hernández, G. (2012). Desempeño y profesionalización: las maestras de párvulos en la historia de la educación en Chihuahua, México, durante las primeras décadas del siglo XX. Historia Caribe, 147157. 
REVISTA DE INVESTIGACIÓN EDUCATIVA DE LA REDIECH N. 10

\section{ISSN: 2007-4336}

Lau, R. (1992). Historia general de Chihuahua. (Vol. 3). Ciudad Juárez: Universidad Autónoma de Ciudad Juárez.

Mayer, A. (2007). México en tres momentos: 1810-19102010. Hacia la conmemoración del Bicentenario de la Independencia y del Centenario de la Revolución Mexicana. retos y perspectivas. México: Universidad Nacional Autónoma de México.

Orozco, V. (1992). Las Guerras indias en la historia de Chihuahua. Primeras fases. México: Consejo Nacional para la Cultura y las Artes.
Ponce de Leon, J. (1907). Datos geográficos y estadísticos del estado de Chihuahua. Chihuahua: G. a: de la Garza.

Robles, M. (1997). Educación y sociedad en la historia de México. México: Siglo XXI editores.

Sandoval, F. (2003). La escuela Modelo, microhistoria de un centro escolar. Chihuahua: Instituto Chihuahuense de la Cultura.

Sociedad Filomática de Chihuahua, México. (1908). Escuelas incorporadas para párvulos, niños y niñas. Chihuahua: Taller "El Norte". 